\title{
Central limit theorem for stationary linear processes generated by linearly negative quadrant-dependent sequence
}

Jiangfeng Wang ${ }^{*}$ and Qunying Wu

* Correspondence: yuanzhang987@163.com College of Science, Guilin University of Technology, Guilin 541004, P. R. China

\begin{abstract}
A central limit theorem is obtained for stationary linear process of the form $X_{t}=\sum_{j=0}^{\infty} a_{j} \varepsilon_{t-j}$, where $\left\{\varepsilon_{i}\right\}$ is strictly stationary sequence of linearly negative quadrant dependent random variables with $E \varepsilon_{i}=0, E\left|\varepsilon_{i}\right|^{s}<\infty$ for some $s>2$, and $\sum_{j=0}^{\infty}\left|a_{j}\right|<\infty$.
\end{abstract}

Keywords: central limit theorem, functional central limit theorem, linearly negative quadrant dependent, linear process

\section{Introduction and main results}

Lehmann [1] introduced a new definition of negative dependence: negative quadrant dependent sequence (NQD). A concept stronger than NQD was introduced by Newman [2] that is said to be linear negative quadrant dependent (LNQD).

Definition 1.1. (Cf. Lehmann [1]). Two random variables $X$ and $Y$ are said to be negative quadrant dependent (NQD, in short) if for any $x, y \in \mathbb{R}$,

$$
P(X<x, Y<y) \leq P(X<x) P(Y<y) .
$$

A sequence $\left\{X_{n}\right\}_{n \in \mathbb{N}}$ of random variables is said to be pairwise NQD, if $X_{i}$ and $X_{j}$ are NQD for all $i, j \in \mathbb{N}^{+}$and $i \neq j$.

Definition 1.2. (Cf. Newman [2]). A sequence $\left\{X_{n}\right\}_{n \in \mathbb{N}}$ of random variables is said to be linearly negative quadrant dependent (LNQD, in short) if for any disjoint subsets $A, B \in \mathbb{Z}^{+}$and positive $r_{j}^{\prime} s$,

$$
\sum_{i \in A} r_{i} X_{i} \text { and } \sum_{j \in B} r_{j} X_{j} \quad \text { are NQD. }
$$

Remark 1.3. It is easily seen that if $\left\{X_{n}\right\}_{n \in \mathbb{N}}$ is a sequence of LNQD random variables, then $\left\{a X_{n}+b\right\}_{n \in \mathbb{N}}$ is still a sequence of LNQD random variables, where $a$ and $b$ are real numbers.

Example 1. Consider three discrete random variables with joint density, $P(x, y, z)=$ $P\left(X_{1}=x, X_{2}=y, X_{3}=z\right)$ with $P(1,3,1)=P(2,3,1)=P(3,2,1)=P(3,3,1)=1 / 11$, $P(3,3,2)=7 / 11$. We could show that $\left(X_{1}, X_{2}, X_{3}\right)$ is LNQD sequence.

(c) 2012 Wang and Wu; licensee Springer. This is an open access article distributed under the terms of the Creative Commons Attribution License (http://creativecommons.org/licenses/by/2.0), which permits unrestricted use, distribution, and reproduction in any medium, provided the original work is properly cited. 
Note that some applications for LNQD sequence have been found. See for example, Newman [2] established the central limit theorem for a strictly stationary LNQD process. Wang and Zhang [3] provided uniform rates of convergence in the central limit theorem for LNQD sequence. Ko and Choi [4] obtained the Hoeffding-type inequality for LNQD sequence. $\mathrm{Fu}$ and $\mathrm{Wu}$ [5] studied the almost sure central limit theorem for LNQD sequences, and so forth.

The aim of this article is to establish a central limit theorem for stationary linear process generated by LNQD random variables.

Let $\left\{X_{n}\right\}_{n \in \mathbb{N}}$ be a stationary process of the form below defined on a probability space $(\Omega, \mathcal{F}, P)$

$$
X_{t}=\sum_{j=0}^{\infty} a_{j} \varepsilon_{t-j},
$$

where $\left\{a_{j}\right\}$ is a sequence of real numbers with $\sum_{j=0}^{\infty}\left|a_{j}\right|<\infty$ and $\left\{\varepsilon_{i}\right\}$ is a strictly stationary sequence such that $E \varepsilon_{i}=0, E \varepsilon_{i}^{2}<\infty$.

The linear processes are of special importance in time series analysis and they arise in wide variety of concepts (see, e.g., Hannan [[6], Chap. 6]). Applications to economics, engineering, and physical science are extremely broad and a vast amount of literature is devoted to the study of the theorems for linear processes under various conditions on $\varepsilon_{t}$. For the linear processes, Fakhre-Zakeri and Lee [7] and Fakhre-Zakeri and Farshidi [8] established CLT under the i.i.d. assumption on $\varepsilon_{t}$ and Fakhre-Zakeri and Lee [9] proved a FCLT under the strong mixing condition on $\varepsilon_{t}$. After Birkel [10] gave Lemma 3 which could be used to prove an inequality the same as the form of Doob's maximal inequality, Kim and Baek [11] obtained a central limit theorem for stationary linear processes generated by linearly positively quadrant dependent process. Peligrad and Utev [12] established the central limit theorem for linear processes with dependent innovations including martingales and mixingale.

Let $S_{n}=\sum_{j=0}^{\infty} X_{j}, \tau^{2}=\sigma^{2} \sum_{j=0}^{\infty}\left(a_{j}\right)^{2}$. Define for $n \geq 1$ the stochastic process

$$
\xi_{n}(u)=n^{-1 / 2} \tau^{-1} S_{[n u]}, \quad u \in[0,1],
$$

where $[x]$ is the greatest integer not exceeding $x$.

Since LNQD is much weaker than NA, and NA is much weaker than independent case, studying the central limit theorems for LNQD fields is of interest. In this article, we establish a CLT (FCLT) for strictly stationary linear process of the form (1.4), generated by an LNQD sequence $\left\{\varepsilon_{i}\right\}$. More precisely, we will prove the following theorems.

Theorem 1.4. Let $\left\{X_{t}\right\}$ be a stationary linear process of the form (1.3), where $\left\{a_{j}\right\}$ is a sequence of constants with $\sum_{j=0}^{\infty}\left|a_{j}\right|<\infty$ and $\left\{\varepsilon_{i}\right\}$ is a strictly stationary LNQD sequence with $E \varepsilon_{i}=0$. Assume

$$
\begin{aligned}
& 0<\sigma^{2}=E \varepsilon_{1}^{2}+2 \sum_{i=2}^{\infty} E\left(\varepsilon_{1} \varepsilon_{i}\right)<\infty, \\
& E\left|\varepsilon_{i}\right|^{s}<\infty \text { for some } s>2 .
\end{aligned}
$$


Then the linear process $\left\{X_{t}\right\}$ fulfills the CLT.

Example 2. Let $X_{t}=\sum_{j=0}^{\infty}\left|a_{j}\right| \varepsilon_{t-j}$ be a strictly stationary linear process generated by LNQD sequence, where $\varepsilon_{t}$ is a strictly stationary LNQD sequence random variables with $E \varepsilon_{t}=0$ and $E\left|\varepsilon_{t}\right|^{\mathrm{s}}<\infty$ for some $s>2$ satisfying (1.5) and (1.6). Here we suppose that the linear operators $a_{j}$ are geometrically bounded, in the sense that there exist real constants $b>0$ and $0<\rho<1$ such that $a_{j} \leq b \rho^{j}$ for all $j>0$. Then this process satisfies Theorem 1.4.

Theorem 1.5. Let $\left\{X_{t}\right\}$ be a stationary linear process of the form (1.3), defined in Theorem 14. If (1.5) and (1.6) are fulfilled, then the process $\left\{\xi_{n}\right\}$ satisfies the FCLT; that is, the process $\left\{\xi_{n}\right\}$ converges weakly to Wiener measure $W$ on the space of all functions on $[0,1]$, which have left-hand limits and are continuous from the right.

\section{Proofs}

The following lemma is needed to prove Theorems 1.4 and 1.5 and it is established by modifying the proof of Lemma 3 in Fakhre-Zakeri and Lee [9]. Doob's maximal inequality has played an important role in their proof. However, in our case, Doob's maximal inequality cannot be used, instead the inequality $E\left(\max _{1 \leq k \leq n}\left|\varepsilon_{1}+\ldots+\varepsilon_{k}\right|^{\prime}\right) \leq$ $B n^{r / 2}$ for $r>2$ and $B$ as in Lemma 2.2 will be used.

Lemma 2.1. Let $\left\{\varepsilon_{i}\right\}$ be a strictly stationary LNQD sequence with mean zero and $0<E \varepsilon_{i}^{2}<\infty . \quad$ Let $\quad X_{t}=\sum_{j=0}^{\infty} a_{j} \varepsilon_{t-j}, \quad S_{k}=\sum_{t=1}^{k} X_{t}, \quad \tilde{X}_{t}=\left(\sum_{j=0}^{\infty} a_{j}\right) \varepsilon_{t}, \quad$ and let $\tilde{S}_{k}=\sum_{t=1}^{k} \tilde{X}_{t}$, where $\left\{a_{j}\right\}$ is a sequence of real numbers with $\sum_{j=0}^{\infty}\left|a_{j}\right|<\infty$. If (1.5) and (1.6) are fulfilled, then

$$
\left(n^{-1 / 2}\right) \max _{1 \leq k \leq n}\left|\tilde{S}_{k}-S_{k}\right| \stackrel{P}{\rightarrow} 0 .
$$

We close this section by introducing a maximal inequality which is needed to prove Lemma 2.1.

Lemma 2.2. Let $\left\{\varepsilon_{t}\right\}$ be a strictly stationary LNQD sequence with mean zero and $0<E \varepsilon_{i}^{2}<\infty$. Assume that (1.6) and (1.7) hold. Then there exist $r>2$ and a constant $B$ not depending on $n$ such that for $n \geq 1$

$$
E\left(\max _{1 \leq k \leq n}\left|\varepsilon_{1}+\cdots+\varepsilon_{k}\right|^{r}\right) \leq B n^{r / 2} .
$$

According to Lemma 3.4 of Zhang [13], we can easily obtain the result.

Proof of Lemma 2.1. As in the proof of Lemma 3 of Fakhre-Zakeri and Lee [9], we have

$$
\begin{aligned}
\tilde{S}_{k} & =\sum_{t=1}^{k}\left(\sum_{j=0}^{k-t} a_{j}\right) \varepsilon_{t}+\sum_{t=1}^{k}\left(\sum_{j=k-t+1}^{k-t} a_{j}\right) \varepsilon_{t} \\
& =\sum_{t=1}^{k}\left(\sum_{j=0}^{t-1} a_{j} \varepsilon_{t-j}\right)+\sum_{t=1}^{k}\left(\sum_{j=k-t+1}^{k-t} a_{j}\right) \varepsilon_{t} .
\end{aligned}
$$

Thus

$$
\begin{aligned}
\tilde{S}_{k}-S_{k} & =-\sum_{t=1}^{k}\left(\sum_{j=t}^{\infty} a_{j} \varepsilon_{t-j}\right)+\sum_{t=1}^{k}\left(\sum_{j=k-t+1}^{k-t} a_{j}\right) \varepsilon_{t} \\
& :=I+I I .
\end{aligned}
$$


It suffices to prove

$$
\left(n^{-1 / 2}\right) \max _{1 \leq k \leq n}|I| \stackrel{P}{\rightarrow} 0,
$$

and

$$
\left(n^{-1 / 2}\right) \max _{1 \leq k \leq n}|I I| \stackrel{P}{\rightarrow} 0 .
$$

First, by Minkowski's inequality and Lemma 2.2 we have for $r>2$

$$
\begin{aligned}
n^{-r / 2} E \max _{1 \leq k \leq n}\left|\sum_{t=1}^{k} \sum_{j=t}^{\infty} a_{j} \varepsilon_{t-j}\right|^{r} & =n^{-r / 2} E \max _{1 \leq k \leq n}\left|\sum_{j=1}^{\infty} \sum_{t=1}^{j \wedge k} a_{j} \varepsilon_{t-j}\right|^{r} \\
& \leq n^{-r / 2}\left(\sum_{j=1}^{\infty}\left|a_{j}\right|\left\{\max _{1 \leq k \leq n}\left|\sum_{t=1}^{j \wedge k} a_{j} \varepsilon_{t-j}\right|^{r}\right\}^{1 / r}\right)^{r} \\
& \leq n^{-r / 2}\left(\sum_{j=1}^{\infty}\left|a_{j}\right| B^{1 / r}(j \wedge n)^{1 / 2}\right)^{r} \\
& =n^{-r / 2}\left(\sum_{j=1}^{\infty}\left|a_{j}\right| B^{1 / r}(j \wedge n)^{1 / 2}\right)^{r} \\
& =\left(\sum_{j=1}^{\infty}\left|a_{j}\right| B^{1 / r}((j \wedge n) / n)^{1 / 2}\right)^{r} \\
& =o(1) .
\end{aligned}
$$

Hence (2.5) is proved by the Markov inequality. To prove (2.6), write $I I=I I_{k 1}+I I_{k 2}$, where $I I_{k 1}=a_{1} \varepsilon_{k}+a_{2}\left(\varepsilon_{k}+\varepsilon_{k-1}\right)+\ldots+a_{k}\left(\varepsilon_{k}+\ldots+\varepsilon_{1}\right)$ and $I I_{k 2}=\left(a_{k+1}+a_{k+2}+\ldots\right)$ $\left(\varepsilon_{k}+\ldots+\varepsilon_{1}\right)$, and let $\left\{p_{n}\right\}$ be a sequence of positive integers such that

$$
p_{n} \rightarrow \infty \text { and } p_{n} / n \rightarrow 0 \text { as } n \rightarrow \infty \text {. }
$$

Then

$$
\begin{aligned}
n^{-1 / 2} \max _{1 \leq k \leq n}\left|I I_{k 2}\right| \leq & \left(\sum_{j=1}^{\infty}\left|a_{j}\right|\right) n^{-1 / 2} \max _{1 \leq k \leq p_{n}}\left|\varepsilon_{k}+\cdots+\varepsilon_{1}\right| \\
& +\left(\sum_{j>p_{n}}\left|a_{j}\right|\right) n^{-1 / 2} \max _{1 \leq k \leq p_{n}}\left|\varepsilon_{k}+\cdots+\varepsilon_{1}\right| \\
:= & I I I+I V .
\end{aligned}
$$

It follows from (2.2), (2.8), that for $r>2$ and a constant $B_{1}$,

$$
\begin{aligned}
& \left(\sum_{j=1}^{\infty}\left|a_{j}\right|\right)^{r} n^{-r / 2} \max _{1 \leq k \leq p_{n}}\left|\varepsilon_{k}+\cdots+\varepsilon_{1}\right|^{r} \\
& \leq\left(\sum_{j=1}^{\infty}\left|a_{j}\right|\right)^{r} B_{1}\left(p_{n} / n\right)^{r / 2}=o(1) .
\end{aligned}
$$


and thus $I I I \stackrel{P}{\rightarrow} 0$ by the Markov inequality. Similarly, by assumption $\sum_{j=1}^{\infty}\left|a_{j}\right|<\infty$ for $r>2$ and a constant $B_{2}$,

$$
\begin{aligned}
& \left(\sum_{j>p_{n}}\left|a_{j}\right|\right)^{r} n^{-r / 2} \max _{1 \leq k \leq p_{n}}\left|\varepsilon_{k}+\cdots+\varepsilon_{1}\right|^{r} \\
& \leq\left(\sum_{j>p_{n}}\left|a_{j}\right|\right)^{r} B_{2}=o(1) .
\end{aligned}
$$

and thus $I V \stackrel{P}{\rightarrow} 0$ by the Markov inequality. Hence, $n^{-1 / 2} \max _{1 \leq k \leq n}\left|I_{k 2}\right| \stackrel{P}{\rightarrow} 0$. It remains to show that $L_{n}=n^{-1 / 2} \max _{1 \leq k \leq n}\left|I I_{k 1}\right| \stackrel{P}{\rightarrow} 0$. For each $m \geq 1$, define $I I_{k 1, m}=$ $b_{1} \varepsilon_{k}+b_{2}\left(\varepsilon_{k}+\varepsilon_{k-1}\right)+\ldots+b_{k}\left(\varepsilon_{k}+\ldots+\varepsilon_{1}\right)$, where $b_{k}=a_{k}$ for $k \leq n$ and $b_{k}=0$ otherwise, and let $L_{n, m}=n^{-1 / 2} \max _{1 \leq k \leq n}\left|I I_{k 1, m}\right|$. Then

$$
L_{n, m} \leq n^{-1 / 2}\left(\left|a_{1}\right|+\cdots+\left|a_{m}\right|\right)\left(\left|\varepsilon_{1}\right|+\cdots+\left|\varepsilon_{m}\right|\right) \stackrel{P}{\rightarrow} 0 \text { as } n \rightarrow \infty .
$$

for each $m$, and

$$
\left|L_{n, m}-L_{n}\right| \leq n^{-1 / 2} \max _{1 \leq k \leq n}\left|\sum_{i=1}^{k}\left(a_{i}-b_{i}\right)\left(\varepsilon_{k}+\cdots+\varepsilon_{k-i+1}\right)\right| .
$$

Since

$$
\left|\sum_{i=1}^{k}\left(a_{i}-b_{i}\right)\left(\varepsilon_{k}+\cdots+\varepsilon_{k-i+1}\right)\right|=\left\{\mid \begin{array}{lr}
0, & k \leq m, \\
\sum_{i=m+1}^{k} a_{i}\left(\varepsilon_{k}+\cdots+\varepsilon_{k-i+1}\right) \mid & \text { otherwise, }
\end{array}\right.
$$

the right-hand side of (2.13) is

$$
\begin{aligned}
& \leq n^{-1 / 2} \max _{m<k \leq n}\left(\sum_{i=m+1}^{k}\left|a_{i}\right|\left|\varepsilon_{k}+\cdots+\varepsilon_{k-i+1}\right|\right) \\
& \leq n^{-1 / 2} \max _{m<k \leq n} \sum_{i=m+1}^{k}\left|a_{i}\right| \max _{m<i \leq k}\left|\varepsilon_{k}+\cdots+\varepsilon_{k-i+1}\right| \\
& \leq n^{-1 / 2} \sum_{i>m}\left|a_{i}\right| \max _{m<k \leq n} \max _{m<i \leq k}\left(\left|\varepsilon_{i}+\cdots+\varepsilon_{k}\right|+\left|\varepsilon_{1}+\cdots+\varepsilon_{k-i}\right|\right) \\
& \leq n^{-1 / 2} \sum_{i>m}\left|a_{i}\right|\left(\max _{m<k \leq n}\left|\varepsilon_{1}+\cdots+\varepsilon_{k}\right|+\max _{m<k \leq n} \max _{m<i \leq k}\left|\varepsilon_{1}+\cdots+\varepsilon_{k-i}\right|\right) \\
& \leq n^{-1 / 2} \sum_{i>m}\left|a_{i}\right|\left(\max _{1 \leq j \leq n}\left|\varepsilon_{1}+\cdots+\varepsilon_{j}\right|+\max _{1 \leq j \leq n}\left|\varepsilon_{1}+\cdots+\varepsilon_{j}\right|\right) \\
& =2 n^{-1 / 2} \sum_{i>m}\left|a_{i}\right| \max _{1 \leq j \leq n}\left|\varepsilon_{1}+\cdots+\varepsilon_{j}\right| .
\end{aligned}
$$

Therefore, it follows from (2.2), (2.12), (2.13) and the Markov inequality that for any $\delta>0$, 


$$
\begin{aligned}
& \lim _{m \rightarrow \infty} \lim _{n \rightarrow \infty} \sup P\left(\left|L_{n, m}-L_{n}\right|>\delta\right) \\
& \leq \lim _{m \rightarrow \infty} 2^{r} \delta^{-r}\left(\sum_{j>m}\left|a_{j}\right|\right)^{r} \lim _{n \rightarrow \infty} \sup n^{-r / 2} E \max _{1 \leq j \leq n}\left|\varepsilon_{1}+\cdots+\varepsilon_{j}\right|^{r} \\
& \leq B \lim _{m \rightarrow \infty} 2^{r} \delta^{-r}\left(\sum_{j>m}\left|a_{j}\right|\right)^{r} \\
& =0 .
\end{aligned}
$$

In view of (2.16) and (2.15), it follows from Theorem 4.2 Billingsley [[14], p. 25].

Proof of Theorem 1.4. As in Lemma 2.1, set $\tilde{X}_{t}=\left(\sum_{j=0}^{\infty} a_{j}\right) \varepsilon_{t}$, and $\tilde{S}_{n}=\sum_{t=1}^{n} \tilde{X}_{t}=\left(\sum_{j=0}^{\infty} a_{j}\right) \sum_{t=1}^{n} \varepsilon_{t}$. Then by (1.4), we have

$$
\begin{aligned}
E \tilde{X}_{t}^{2}+2 \sum_{t=2}^{\infty} E\left(\tilde{X}_{1} \tilde{X}_{t}\right) & =\left(\sum_{j=0}^{\infty} a_{j}\right)^{2} E \varepsilon_{1}^{2}+2\left(\sum_{j=0}^{\infty} a_{j}\right)^{2} \sum_{t=2}^{\infty} E\left(\varepsilon_{1} \varepsilon_{t}\right) \\
& =\left(\sum_{j=0}^{\infty} a_{j}\right)^{2} \sigma^{2}=\tau^{2}<\infty .
\end{aligned}
$$

Since $\tilde{X}_{t}^{\prime} s$ form a stationary LNQD sequence, $\left\{\tilde{X}_{t}\right\}_{t \in \mathbb{N}}$ satisfies the CLT by Theorem 12 of Newman [2]; that is,

$$
n^{-1 / 2} \tilde{S}_{n} \stackrel{D}{\rightarrow} N\left(0, \tau^{2}\right)
$$

According to Lemma 2.1, we also have

$$
n^{-1 / 2}\left|\tilde{S}_{n}-S_{n}\right| \stackrel{P}{\rightarrow} 0 .
$$

Hence from (2.18) and (2.19) the desired conclusion follows.

Proof of Theorem 1.5. Note that $\left\{\tilde{X}_{t}\right\}$ is a stationary LNQD process and that,

$$
E\left|\tilde{X}_{t}\right|^{s}=E\left|\left(\sum_{j=0}^{\infty} a_{j}\right) \varepsilon_{t}\right|^{s}=\left|\sum_{j=0}^{\infty} a_{j}\right|^{s} E\left|\varepsilon_{t}\right|^{s} \leq\left(\sum_{j=0}^{\infty}\left|a_{j}\right|\right)^{s} E\left|\varepsilon_{t}\right|^{s} .
$$

Thus it follows from Lemma 2.2 and (2.20), that $\left\{\tilde{X}_{t}\right\}$ satisfies the conditions of Corollary 2 of Birkel [10]. This implies that Theorem 1.5 holds for the sequence $\left\{\tilde{\xi}_{n}\right\}$, where we define $\tilde{\xi}_{n}$ as in (1.4), with $\tilde{S}_{r}$ replacing $S_{r}$. By Lemma 2.1, $\left|\tilde{\xi}_{n}(u)-\xi_{n}(u)\right| \stackrel{P}{\rightarrow} 0$ for all $0 \leq u \leq 1$. Hence, the desired conclusion follows.

\section{Acknowledgements}

This study was supported by the National Natural Science Foundation of China (11061012), the Guangxi China Science Foundation (2011GXNSFA018147) and Innovation Project of Guangxi Graduate Education (2010105960202M32). We are very grateful to the referees and the editors for their valuable comments and some helpful suggestions that improved the clarity and readability of the paper.

\section{Authors' contributions}

QW participated in the design of the study. JW conceived of the study, and participated in the design and proof of the article. All authors read and approved the final manuscript. 


\section{Competing interests}

The authors declare that they have no competing interests.

Received: 7 June 2011 Accepted: 28 February 2012 Published: 28 February 2012

\section{References}

1. Lehmann, EL: Some concepts of dependence. Ann Math Stat. 37, 1137-1153 (1966). doi:10.1214/aoms/1177699260

2. Newman, CM: Asymptotic independence and limit theorems for positively and negatively dependent random variables. In: Tong, YL (eds.) Statistics aand probability. 5, 127-140 (1984)

3. Wang, J, Zhang, L: A Berry-Esseen theorem for weakly negatively dependent random variables and its applications. Acta Math Hung. 110(4), 293-308 (2006). doi:10.1007/s10474-006-0024-x

4. Ko, MH, Choi, YK, Choi, YS: Exponential probability inequality for linearly negative quadrant dependent random variables. Korean Math Soc Commun. 22, 137-143 (2007). doi:10.4134/CKMS.2007.22.1.137

5. $\mathrm{Fu}, \mathrm{YL}, \mathrm{Wu}, \mathrm{QY}$ : Almost sure central limit theorem for LNQD sequences. J Guilin Univ Technol. 30(4), 637-639 (2010)

6. Hannan, EJ: Multivariate Time Series. Wiley, New York (1970)

7. Fakhre-Zakeri, I, Lee, S: Sequential estimation of the mean of a linear process. Sequential Anal. 11, 181-197 (1992). doi:10.1080/07474949208836252

8. Fakhre-Zakeri, I, Farshidi, J: A central limit theorem with random indices for stationary linear processes. Statist Probab Lett. 17, 91-95 (1993). doi:10.1016/0167-7152(93)90002-Z

9. Fakhre-Zakeri, I, Lee, S: A random functional central limit theorem for stationary linear processes generated by matingales. Statist Probab Lett. 35, 417-422 (1997). doi:10.1016/S0167-7152(97)00040-0

10. Birkel, P: A functional central limit theorem for positively dependent random variables. J Multi Anal. 44, 314-320 (1993). doi:10.1006/jmva.1993.1018

11. Kim, TS, Baek, Jl: A central limit theorem for stationary linear processes generated by linearly positively quadrantdependent process. Statist Probab Lett. 51, 299-305 (2001). doi:10.1016/S0167-7152(00)00168-1

12. Peligrad, M, Utev, S: A entral limit theorem for stationary linear processes. Ann Probab. 34(2), 1608-1622 (2006)

13. Zhang, LX: A functional central limit theorem for asymptotically negatively dependent random fields. Acta Math Hung. 86, 237-259 (2000). doi:10.1023/A:1006720512467

14. Billingsley, P: Convergence of Probability Measures. Wiley, New York (1968)

doi:10.1186/1029-242X-2012-45

Cite this article as: Wang and Wu: Central limit theorem for stationary linear processes generated by linearly negative quadrant-dependent sequence. Journal of Inequalities and Applications 2012 2012:45.

\section{Submit your manuscript to a SpringerOpen ${ }^{\circ}$} journal and benefit from:

- Convenient online submission

Rigorous peer review

- Immediate publication on acceptance

- Open access: articles freely available online

- High visibility within the field

- Retaining the copyright to your article

Submit your next manuscript at $\gg$ springeropen.com 